\section{Las fibras vegetales: materiales ancestrales para un futuro sostenible en el desarrollo de productos}

Juan Manuel España Espinoza *

Resumen: Latinoamérica se caracteriza por la riqueza biótica y la biodiversidad de sus ecosistemas que desde épocas prehispánicas fueron el sostén de los asentamientos humanos de todo el continente. Esta diversidad favoreció el surgimiento de comunidades indígenas caracterizadas por el territorio, que les proporcionaba lo que requerían para su sustento y desarrollo. Las fibras vegetales han sido desde esas épocas un factor de identidad y un reflejo de riqueza cultural material e inmaterial. Lazos, vestimentas, aperos, mochilas y demás objetos de las etnias indígenas y de la cultura campesina latinoamericana son reflejo de saberes. La invasión de los polímeros derivados del petróleo relego y amenazó la continuidad en el uso de las fibras vegetales. Las botas plásticas, los lazos de polipropileno, los mantos y telas de poliéster pusieron por décadas a las fibras vegetales en un segundo plano y propiciaron la desaparición de saberes ancestrales manifiestos en artesanía utilitaria.

Sin embargo, el amenazante incremento del calentamiento global, la contaminación de recursos hídricos con plásticos y las enormes consecuencias en los ecosistemas a causa nuestra demanda por el petróleo y sus derivados, ha llevado al mundo en las últimas décadas a fortalecer la investigación y desarrollo en nuevos materiales y aplicaciones de las fibras vegetales y los subproductos de la agroindustria, para reemplazar o minimizar el uso de los plásticos.

Ahora es Latinoamérica una de las regiones con más potencial de bioprospección en el uso y aprovechamiento de fibras vegetales, tanto por la abundancia y diversidad que posee como por los saberes, técnicas y conocimientos que están vinculados con las comunidades rurales de todo el continente. Sin embargo, pareciera que nos estamos rezagando en la investigación alrededor de las fibras y su potencial en nuevos materiales y aplicaciones. ¿Qué fibras son mejores en el desarrollo de materiales compuestos?, ¿cuáles son las ventajas y consecuencias ambientales de incrementar su uso y aprovechamiento?, ¿cómo beneficia y/o afecta a las comunidades rurales que producen estas fibras? ¿Cuáles son sus cualidades físicas y mecánicas?, son algunas de las muchas preguntas que deberíamos desde la investigación estar indagando. Y aunque existen diversos procesos investigativos en la academia a este respecto, la revisión de artículos indexados y publicaciones académicas parece indagar que son otras regiones como Europa, Norteamérica y Asia las que están tomando la delantera en la investigación, uso y aplicación de las fibras en nuevos materiales y productos, a pesar de no contar con una biodiversidad como la Latinoamericana. En la Universidad Jorge Tadeo Lozano de Bogotá, desde la Escuela de Diseño de producto estamos enfocados, en investigar en el uso de fibras vegetales y subproductos de la agroindustria para el desarrollo de nuevos materiales y aplicaciones. El diseño tuvo un 
rol muy importante en la masificación del uso de los plásticos, ahora debe ser parte activa en la búsqueda de materiales alternativos que potencien el aprovechamiento sostenible de los recursos y el fortalecimiento de desarrollos de producto para economías locales. Las fibras vegetales fueron en el pasado la cuna de saberes locales para resolver las necesidades cotidianas y rurales, es tiempo que retomemos esta ruta. Un grupo de investigadores conformado por profesores y estudiantes de pregrado y posgrado, en asocio con instituciones de investigación agrícola y comunidades productoras de fibras, estamos en la búsqueda de desarrollar nuevos materiales compuestos $(\mathrm{ACM})^{1}$ biopolímeros y sistemas de transformación tecnológica local, para incentivar el uso y aplicación de materiales locales en el desarrollo de productos capaces de competir con los fabricados en materiales plásticos, pero con todas las ventajas que implica el uso de materiales biodegradables y sostenibles. Este artículo describe la ruta que como investigadores estamos recorriendo e invita a pares de otras latitudes a vincularse con este esfuerzo.

Palabras claves Fibras vegetales - Sostenibilidad - Materiales Plásticos - Saberes ancestrales - Desarrollo tecnológico - Nuevos materiales - ACM.

[Resúmenes en inglés y portugués en las páginas 235-237]

(*) Juan Manuel España Espinoza. Candidato a Doctor en Ciencias Ambientales y Sostenibilidad; Especialista en Gerencia de Diseño, ambas de la Fundación Universidad de Bogotá Jorge Tadeo Lozano. Magister en Gestión ambiental, Diseñador Industrial ambas de la Pontificia Universidad Javeriana. Profesor e Investigador con amplia experiencia en el campo del diseño participativo con énfasis en reflexiones ambientales y sociales. Conocimiento y cercanía con diferentes comunidades y técnicas artesanales, con las que trabaja desde el año 2001. Su principal campo investigativo está conectado la investigación aplicada al sector rural, con fortaleza y conocimiento en el manejo e importancia cultural, económica y ambiental de las fibras naturales como alternativa de desarrollo sostenible y con el desarrollo tecnológico aplicado al sector agrícola, desde enfoques de aprovechamiento integral, economía circular y beneficio social. Investigador principal y coinvestigador de diversos proyectos enfocados en desarrollo tecnológico para el sector agrícola (Ver $\mathrm{CV}$ extenso en Pág 312).

\section{Las fibras naturales, registro viviente del territorio}

Latinoamérica es una región de mucha riqueza natural, la constituyen muchos de los países más biodiversos del mundo, tanto en especies animales como vegetales; esta inmensa riqueza natural fue fundamento en la diversidad cultural prehispánica de aquellos que habitaron el territorio. 
Fibras procedentes de tallos, hojas, frutos y animales fueron material propicio para sogas, lazos, cordeles, hilos, telas, mantos, contenedores, canastos, vestidos, etc. Y tan variados como su procesamiento eran las características de las diversas fibras, sus territorios y las connotaciones sociales, culturales y religiosas de los objetos con ellas concebidos.

La cultura latinoamericana está ligada a la historia en el uso y aplicaciones de las fibras naturales, estas han permitido a grupos humanos de todo el continente, plasmar su identidad cultural y su saber en objetos y tejidos de uso local y cotidiano.

Todas las culturas prehispánicas, Aztecas, Mayas Incas, Guanes, Muiscas, Guayakís, Tzeltales, Aimaras Caribes etc. Tuvieron en las fibras materiales de apoyo y soporte para el desarrollo de una artesanía utilitaria y simbólica. Fueron insumo y cimiento de la creatividad y recursividad.

Un ejemplo de esto lo podemos verlo en Colombia, que no fue ajeno al uso extendido y ancestral de las fibras como material de artesanía utilitaria, país catalogado como uno de los tres más ricos en biodiversidad, poseedor de un amplio abanico de fibras utilizadas por diferentes etnias, extraídas de diversas partes de plantas y animales, con distintos usos y aplicaciones. El trabajo investigativo adelantado por artesanías de Colombia en 2008 detecto 114 plantas usadas, para extraer fibras vegetales, que sin ser un total da una idea de la amplia diversidad existente.

El término fibra vegetal puede hacer alusión a pelos unicelulares que crecen sobre la superficie de las semillas del algodón (Gossypium hirsutum, G. barbadense, G. arboreum, G. herbaceum), a pelos multicelulares extraídos de las hojas del fique (Furcraea cabuya, F. macrophylla), a la médula esponjosa de los tallos del lecho (Rhabdadenia biflora), a las raíces de los chagualos y gaques (Clusia alata, C. crenata, C. lineata, C. multiflora) y el yaré (Heteropsis ecuadorensis, H. fl exuosa, H. linearis, H. oblongifolia, H. spruceana, H. steyermarkii, $\mathrm{H}$. tenuispadix), a los tallos de las chinas y atacorrales (Smilax domingensis, $\mathrm{S}$. tomentosa), a la epidermis de los tallos del chocolatillo (Ischnosiphon arouma, I. infl atus, I. obliquus, I. puberulus), a las brácteas de la inflorescencia del cabecinegro (Manicaria saccifera), a la nervadurade la hoja de la caña flecha (Gyneriumsagittatum), a los cogollos de la iraca (Carludovica palmata) y el gürregue (Astrocaryum standleyanum), a los ejes de las inflorescencias del esparto (Juncus ramboi), a las cortezas de la yanchama (Ficus insipida, F. maxima, F. mutisii, F. schippi) y la damagua (Poulsenia armata), o a toda la planta como en la paja blanca (Calamagrostis effusa, C. intermedia), entre tantas especies empleadas en Colombia (Linares, E.L., Galeano, N. Garcia \& Y, Figueroa, 2008).

La llegada de los españoles altero de manera irreversible muchas de las formas y usos de las fibras naturales, muchos pueblos indígenas fueron aniquilados y otros diezmados, los Guanes por ejemplo, etnia indígena que habitaba la región de Guanentá en Colombia trabajaban de manera extendida el fique ${ }^{3}$ en diversos elementos de uso cotidiano como mochilas ${ }^{4} \mathrm{y}$ contenedores, este pueblo guerrero se enfrentó a los españoles hasta su virtual exterminio, sin embargo la región biogeográfica que habitaban mantiene el legado en el uso del fique hasta el día de hoy. Aunque los españoles de manera violenta borraron mucho del patrimonio indígena, el mismo proceso de mestizaje y las técnicas traídas por los españoles a la vez que sus fibras, diversificaron el abanico técnico en el uso y manejo de estas. Este proceso se replicó en diversos lugares del continente donde las etnias sobrevivientes o sus descendientes, mantuvieron la tradición en el uso de materiales y técnicas 
hibridando con lo traído por los españoles, pero evitando la pérdida del legado y del conocimiento en la transformación del recurso, este conocimiento a su vez se fue arraigando en la cultural campesina popular, el uso de fibras vegetales para el calzado y los utensilios del día a día en el campo se convirtieron en constantes no solo en Colombia sino en muchos países Latinoamericanos.

En otras latitudes, del continente, en países como Bolivia y Perú el uso de la Alpaca ha sido símbolo de los pueblos indígenas, e incluso hoy en día regiones como Puno en Bolivia derivan gran parte de su actividad económica de esta fibra animal. Los datos censales muestran que, en esta región la crianza de alpacas es la fuente de empleo de 185,780 personas que dependen económicamente de esta actividad. Salas, P. (2015). Y en países como Argentina o Uruguay con especies introducidas por la conquista como las ovejas se formaron focos de identidad y desarrollo rural campesino, a la par con un saber hacer con las fibras de dichos animales.

A principios del siglo 19 las fibras naturales empiezan a ser parte de la revolución industrial, México ve en el Henequén una industria con potencial, e inicia un desarrollo tecnológico amplio y significativo, que podría haber conducido de mantenerse en el tiempo a que México fuese ejemplo de desarrollo y aprovechamiento de fibras naturales. Aunque prácticamente casi cualquier especie y variedad de agave puede ser fuente del ixtle usado en las diferentes artesanías regionales (México) con sus evidentes diferencias en usos y calidad de fibra son dos los casos histórica y culturalmente sobresalientes: el raspado de henequén y el tallado de lechuguilla. Pérez, A. (1999).

Estos ejemplos muestran como a lo largo y ancho del continente las fibras naturales de origen animal y vegetal han sido manifestación histórica, cultural y biótica del territorio. Sin embargo, el objetivo de este artículo no es explorar el pasado, al contrario, busca indagar y reflexionar sobre los potenciales futuros de este legado ancestral.

\section{La actualidad de los materiales de origen petroquímico}

No podemos seguir hablando de fibras sin conversar brevemente de los materiales plásticos y las fibras sintéticas, que con su aparición relegaron a los materiales naturales a un segundo plano convirtiéndose en la principal causa de la desaparición de diversos oficios y productos en fibras vegetales. Alrededor del mundo miles de artesanos de fibras naturales empezaron a ver sus productos desplazados por aquellos elaborados en fibra plástica. España, et, Al. (2015).

El bajo costo de las fibras plásticas, su alta durabilidad y resistencia a las condiciones ambientales, y su adaptación productiva a diversos tipos de sistemas de transformación. La hicieron la materia prima por excelencia de empaques para alimentos, fibras textiles, calzado y un sin numero de productos. Hoy en día el plástico está presente en prácticamente todos los productos de uso cotidiano y se fabrican objetos que compiten con los de fibras naturales en todos los sectores.

En las décadas de la posguerra los polímeros expandieron su uso de modo exponencial, estábamos deslumbrados por su potencial como material, pero ciegos a los impactos ambientales que traería en el futuro cercano y al impacto socio-cultural inmediato en las 
economías locales y artesanales que vieron desplazados sus productos tradicionales de artesanía utilitaria por aquellos de origen industrial producidos con materiales poliméricos. Hoy en día vivimos una hecatombe ambiental de basura plástica, factores, como su baja biodegradabilidad que se veían como ventaja frente a los materiales naturales, están demostrando ser muy problemáticos, sobretodo en los plásticos de un solo uso que están en la mira de las legislaciones ambientales, por su masivo impacto en ecosistemas terrestres y marinos.

Los materiales que puedan reemplazar o disminuir el uso de material plástico, están en siendo de interés para investigadores, empresas y consumidores.

\section{Una oportunidad verde, para las fibras naturales}

Las realidades ambientales actuales, están sensibilizando a los consumidores a escoger productos cuyas características de fabricación y sus materiales cumplan con estándares más exigentes en términos de impacto ambiental, a su vez los gobiernos se están viendo forzados ha tomar decisiones legislativas más estrictas con el uso de ciertos materiales e insumos. Esto esta generando un ambiente propicio para el fortalecimiento de los sectores locales cuyos potenciales productivos puedan generar menores consecuencias medioambientales. Las fibras vegetales llevan décadas en la mira de investigadores, que han analizado y testeado sus características físicas y su rendimiento como materiales aplicables al desarrollo de productos y a usos de tipo arquitectónico entre otros. Las fibras naturales han aumentado su aplicación como complemento a los materiales plásticos, en diversas usos industriales y arquitectónicos sustituyendo principalmente a la fibra de vidrio, (Joshi et al. 2004). La capacidad de las fibras vegetales de mejorar propiedades físicas de la estructura de los objetos como peso, flexibilidad y capacidad de absorción de impactos, han sido demostradas en diversas investigaciones. Los materiales con fibras naturales están surgiendo como alternativa a los compuestos plásticos reforzados con fibra de vidrio (Wambua et al. 2003; Bogoeva-Gaceva et al. 2007; Taj et al. 2007; Zah et al. 2007 John y Thomas 2008; Bavan y Kumar 2010; Mohammed et al. 2015).

Sin embargo en el panorama latinoamericano pareciera que estamos relegados en los procesos de investigación y en el uso innovador y tecnológico de fibras naturales en nuevos productos y aplicaciones, son los países del hemisferio norte junto a China y la India los que estas avanzando a pasos gigantes en este tema. La presencia de fibras vegetales como yute, cáñamo o sisal en materiales compuestos de la industria automotriz es una realidad hace décadas, el uso de estas fibras en la arquitectura, en materiales reforzados, ultraligeros y en un sinfín de usos ya es un proceso avanzado. Y aunque Latinoamérica posee una abundancia biótica y un saber ancestral arraigado y diverso, pareciera que nos estamos relegando en la investigación y desarrollo de nuevos materiales y en ejercicios de bioprospección en este campo. Esto no es solo un tema tecnológico y ambiental, los productores agrícolas de fibras naturales en el continente tendrían una oportunidad de mejorar sus ingresos y condiciones productivas en la medida que los usos y aplicaciones de las fibras se realicen en productos y desarrollos de mejor valor en mercado. En la actualidad muchos productores de fibras venden sus materiales, con nulo o muy bajo nivel de ganancia. En 
Colombia por ejemplo la mayor parte de la fibra de fique que se produce se utiliza en la icónica elaboración de costales de café, los cuales elabora la industria con fibra comprada principalmente a pequeños productores, en la medida que los costales para café deben competir en precio con los plásticos es un producto de bajo valor agregado, lo que se le paga al productor es muy poco, en correlación con todo el esfuerzo productivo y financiero que implica la obtención de la fibra. Se requiere de usos y aplicaciones que vinculen de modo incluyente al productor en esquemas de comercio justo y en aplicaciones y usos de la fibra que presenten mayor valor y reconocimiento en mercado.

En Latinoamérica si existen investigaciones y desarrollo en el área de las fibras vegetales, pero la mayoría parecen estarse quedando en un nivel meramente académico en el cual desde una revisión de producción científica pareciéramos también estar muy por detrás de otras regiones del planeta que no poseen un potencial Biótico tan diverso. Pareciera que en el sector de las fibras naturales una vez más somos exportadores de materias primas, pero no nicho de desarrollo e innovación tecnológica en nuevos materiales y aplicaciones.

\section{Luchando contra la corriente}

Si solo en Colombia se reconocen más de 248 especies de plantas cuyas fibras tienen diversos usos y aplicaciones desde épocas prehispánicas (Linares 1994). Muchas de éstas tienen usos artesanales y/o industriales con un legado sociocultural que impacta todos los departamentos. Actualmente se usan 118 fibras de origen vegetal principalmente en objetos artesanales y de uso cotidiano (Linares et al., 2017). Estas cifras se refieren únicamente a aquellas plantas que tienen un uso ancestral o comprobado. Este mapeo no incluye subproductos del sector agrícola, ni materiales aun no explorados desde el saber vernáculo. Y estamos hablando de solo un país del continente. ¿Cuál es potencial de Latinoamérica en bioprospección de fibras naturales? Ni siquiera las fibras de uso artesanal reconocido están caracterizadas y estudiadas en su potencial para el desarrollo de nuevos materiales y productos.

Pero en este apartado quiero aclarar que si existen investigadores e instituciones que con pocos recursos y con muchas dificultades propias de la investigación en nuestra región, estamos buscando aportar en este campo. Hace poco conocí de primera mano una iniciativa en Argentina adelantada por el INTI ${ }^{5}$ para el desarrollo de nuevos materiales y productos en fibra de lana no tejida de ovejas de carne. Una iniciativa muy interesante que unía el aprovechamiento de una fibra poco utilizada, el proyecto se centra en la lana corta subproducto de la esquila de ovejas de carne, con una técnica de afieltrado, aplicado al desarrollo de nuevos productos y vinculando de manera activa a las comunidades rurales. En una lógica de investigación-acción, tomaron contacto directo con los actores involucrados en la cadena de la lana, se desarrolló un proyecto participativo para estimular su apropiación colectiva, fomentando el desarrollo local. Ariza, Et, al. (2014).

Y como está iniciativa del INTI existen muchos desarrollos en diversos países latinoamericanos, sin embargo, pareciera que nos falta fortalecer las sinergias y los aprendizajes colectivos a la vez que se tejen redes de conocimiento en el campo de las fibras, a fin de compensar nuestras limitaciones en recursos financieros para la investigación. Es difícil 
pensar que, en el corto o mediano plazo, tendremos recursos para la investigación aplicada similares a la Unión Europea o a China, pero si podríamos fortalecer el trabajo colaborativo y el colegie territorial como una forma de fortalecer nuestro potencial en la investigación y desarrollo en las fibras naturales.

\section{Nuestra experiencia}

En la escuela de diseño de producto y el programa de diseño industrial, de la Universidad Jorge Tadeo Lozano de Bogotá, estamos desde el 2012 investigando y trabajando en fibras vegetales, desde tres niveles diferentes, pero ampliamente correlacionados.

Un primer nivel pedagógico que vincula a los estudiantes desde el trabajo de campo y el diseño colaborativo con las comunidades rurales que cultivan y transforman las fibras vegetales. Este proyecto no solo es un mecanismo de aporte a nuestro déficit con el sector agrícola, también es un escenario pedagógico de contextualización y conexión de los estudiantes con una parte de Colombia que, entre las nuevas tecnologías y nuestro desarraigo por el campo, es casi desconocida para la gran mayoría de jóvenes que formamos en nuestras universidades (España et al., 2019).

Un segundo nivel de investigación aplicada, articulada y con cofinanciación de instituciones de investigación agropecuaria como AGROSAVIA ${ }^{6}$. Esta alianza, entre Universidad y estado ha buscado desde un acercamiento a las necesidades sectoriales y un constante dialogo con las comunidades campesinas y artesanas, entender las necesidades del sector y desde ese lugar desarrollar ofertas tecnológicas que ayuden a resolver los retos y necesidades productivas y ambientales. Esta relación ya lleva más de 5 años articulando acciones y desarrollos investigativos colaborativos. Se puede destacar de este proceso el desarrollo tecnológico realizado por las dos instituciones para mejorar el proceso de obtención de la fibra de fique y sus subproductos, para lo cual se desarrollo un prototipo funcional, que permite obtener fibra, jugo y bagazo por separado y para su aprovechamiento.

Y un tercer nivel, que es el que más desarrollare en este artículo, que vinculando los dos anteriores (estudiantes de pregrado, instituciones investigadoras) y el proceso doctoral del autor busca desarrollar nuevos uso y aplicaciones para las fibras naturales. Desde tres frentes distintos:

Utilizando fibras y desperdicios de la agroindustria, se van a desarrollar materiales y sus aplicaciones a productos, acordes a las características físicas de los mismos. Las líneas de desarrollo son (Ver Figura 1):

a. Desarrollar biopolímeros a base de polisacáridos, y fibro-reforzarlos con materiales vegetales como la fibra de fique, la hoja del maíz o la fibra de coco; Y diseñar usos y aplicaciones de baja complejidad tecnológica, para que dicha tecnología sea transferible a sectores rurales.

En la Figura 2, se puede apreciar el grupo de polímeros biodegradables que se vienen estudiando y cuyas formulas de elaboración y de composición ya se están desarrollando y afinando con el equipo investigativo. En este ramo se encuentran almidones de origen agrícola local y carrageninas que se pueden obtener de la biomasa de las algas, cuya pro- 
Desarrollo y adaptación de sistemas de transformación de bajo costo y complejidad tecnológica

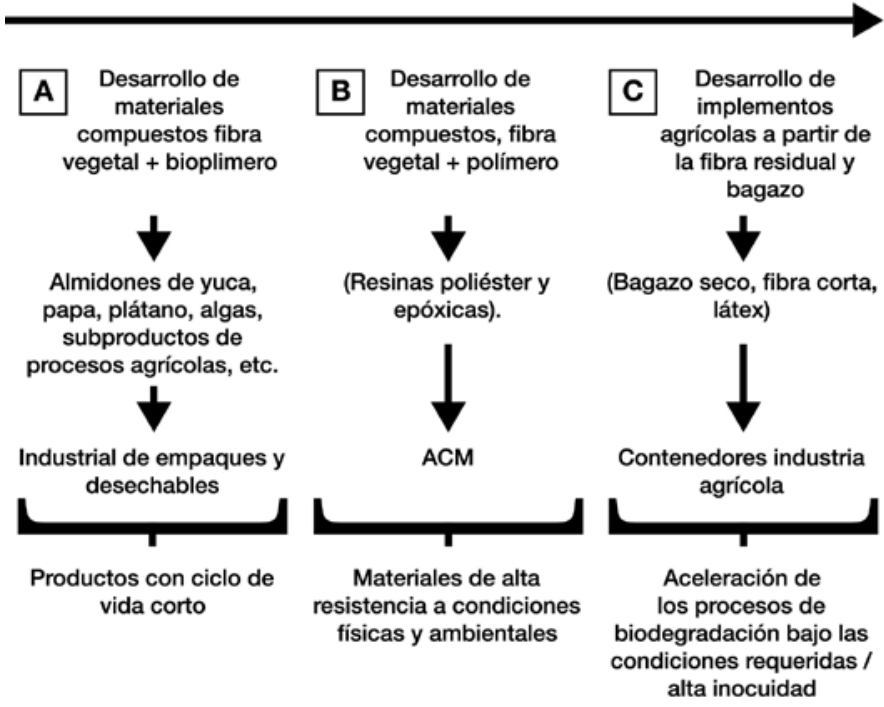

Figura 1. Áreas de intervención investigativa.

liferación en algunas regiones de costa es una problemática ambiental asociable a cambio climático.

Aunque todavía no se han explorado los potenciales usos de los materiales obtenidos se espera a principios del 2019 iniciar pruebas y aplicaciones en el desarrollo de producto. Esta iniciativa investigativa ambiciona conectar investigación básica con aplicabilidad comercial, que beneficie a los sectores rurales.

b. Desarrollar materiales ACM usando fibras naturales no tejidas que reduzcan el uso de plásticos y que reemplacen en materiales compuestos a fibras tan nocivas como la de vidrio. Este es uno de los campos con mayor desarrollo desde la literatura científica, sin embargo, el mapeo de usos y aplicaciones utilizando fibras locales es limitado y la comprensión de las implicaciones ambientales del uso de una fibra u otra poco explorado. Es amplio el potencial a desarrollar en el mapeo de fibras como refuerzo de materiales compuestos.

La Figura 3, esquematiza las rutas de desarrollo que se están planteando junto con AGROSAVIA para el desarrollo y aplicación de materiales fibroreforzados, aunque el cuadro se 


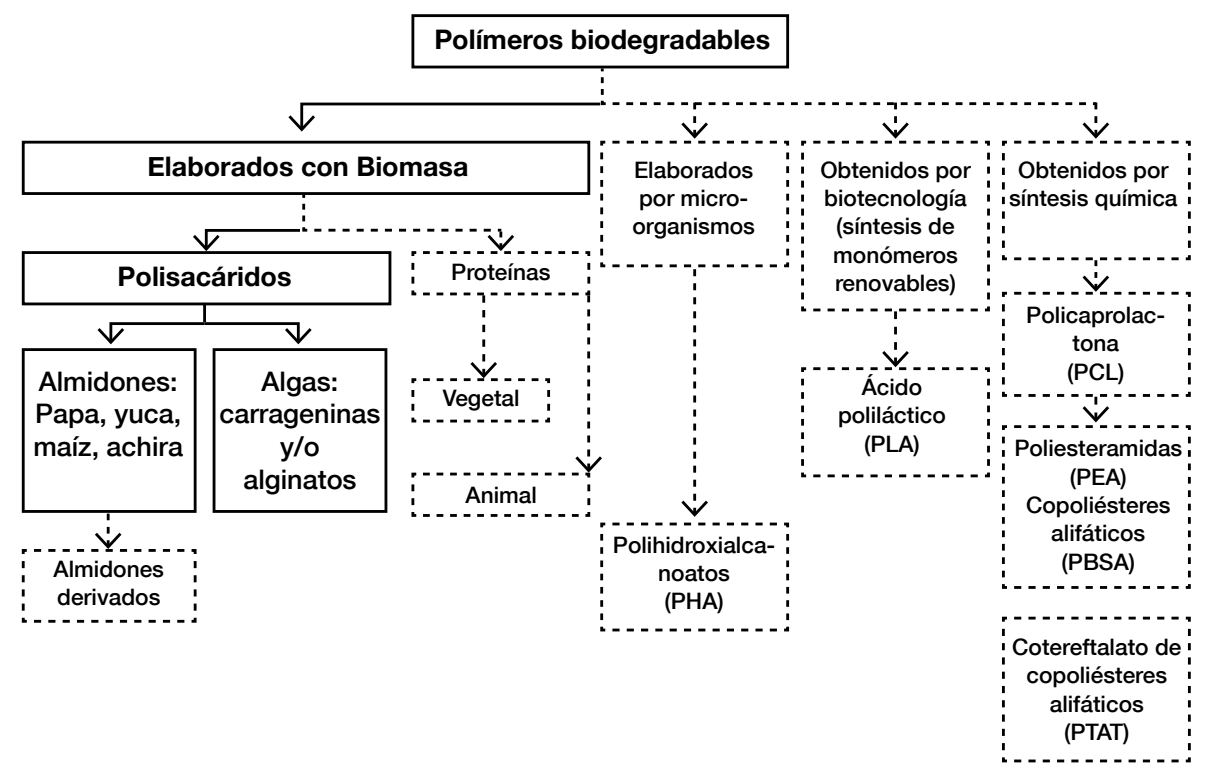

Figura 2. Origen de los polímeros biodegradables. Modificado de Saiter et al, 2012.

centra en el uso de la fibra de fique y de piña, ya se han vinculado a las pruebas de material otros tipos de fibras y compuestos vegetales. Quisiera destacar como el esquema vincula la huella ecológica en el proceso de balance de la relación material-producto y como la investigación no solo está abordando una categorización física del material, en la medida que lleva el desarrollo hasta prototipos y productos.

c. El desarrollo de implementos agrícolas a partir de fibra y bagazo residual, busca hacer sinergia entre los residuos subproducto de la producción agrícola y necesidades especificas de los sectores rurales donde se generan estos subproductos; busca recuperar la autosuficiencia local de suplir algunas necesidades desde una artesanía utilitaria.

En la Figura 4 se puede apreciar una de las primeras metas de esta iniciativa, que es el desarrollo de contenedores para germinar plántulas, y que estas puedan ser transplantadas directamente al suelo sin perjuicio alguno para la planta ni para el medio ambiente. El uso de bolsas plásticas y otros insumos de polímeros, para germinar plantas en el campo es un problema masivo. Que afecta la calidad de los suelos. 


\section{DESARROLLO DE MATERIALES COMPUESTOS A PARTIR DE FIBRAS VEGETALES (Furcraea ssp y Ananas ssp)}

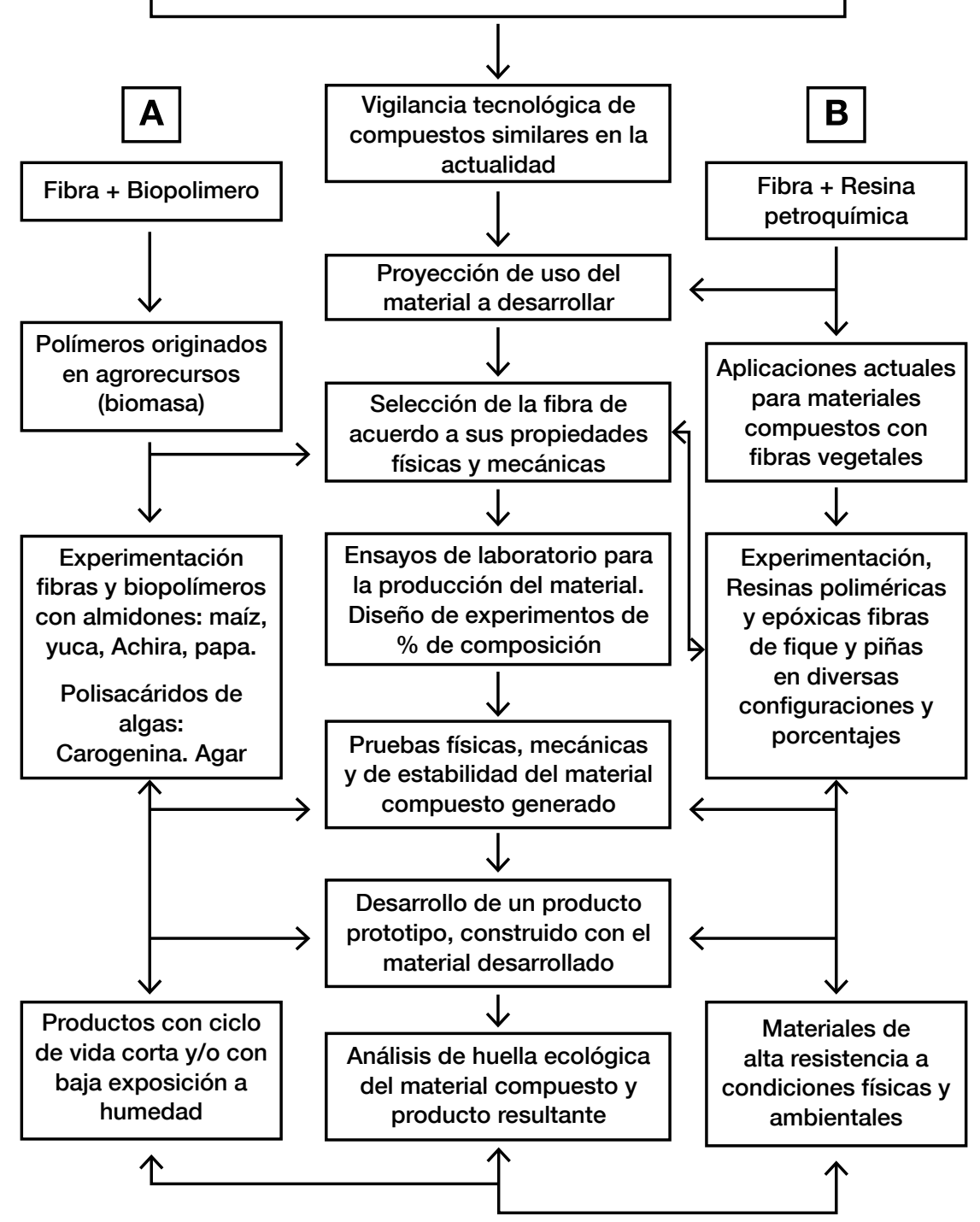

Figura 3. Esquema general de desarrollo de materiales compuestos a partir fibra vegetal+biopolímero (A) y fibra vegetal+polímero de origen petroquímico (elaborada por JM España-UTadeo y E Barbosa-Agrosavia). 


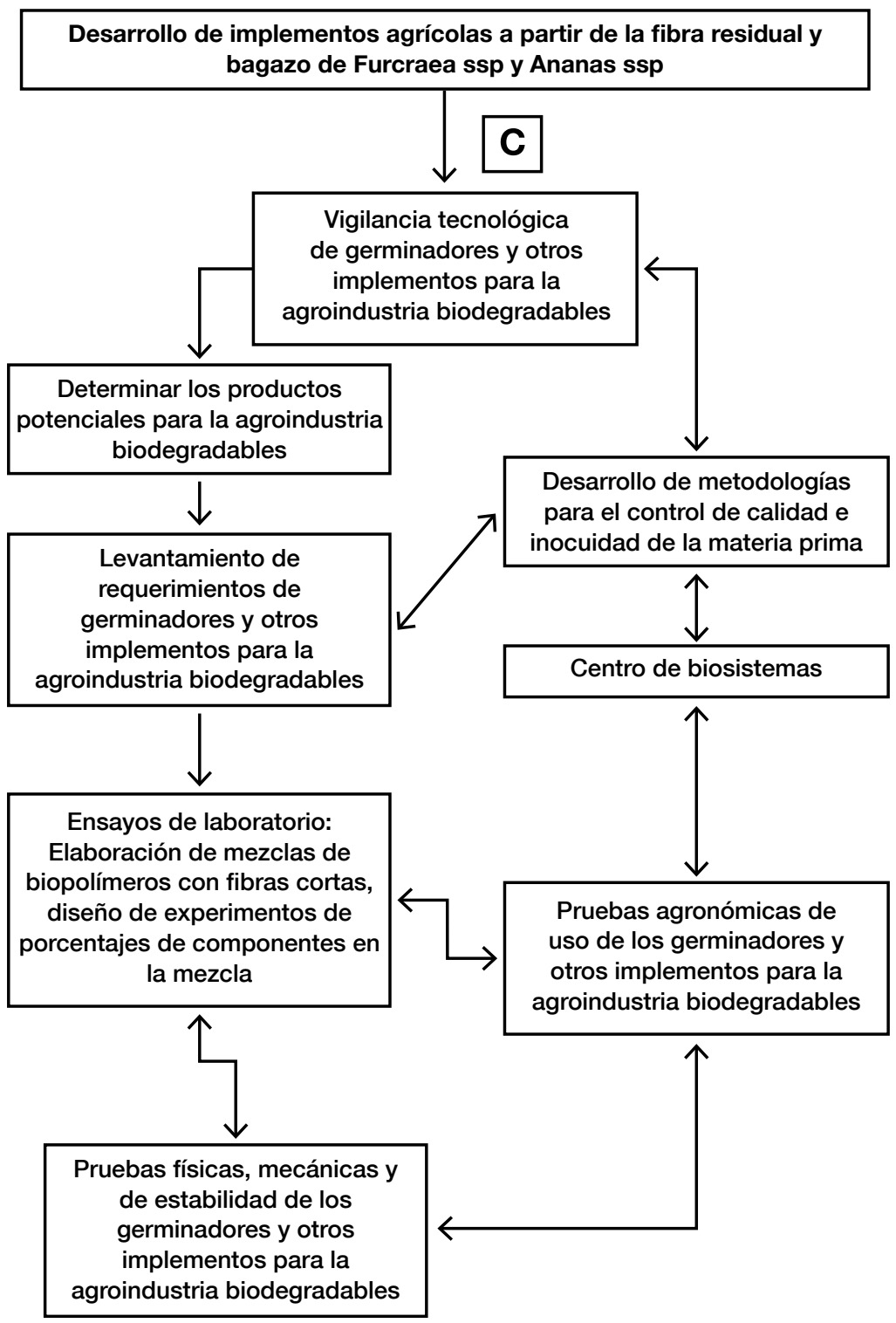

Figura 4. Esquema de aplicación en el desarrollo de implementos agrícolas a partir de fibra residual y bagazo del fique (Furcraea spp.) y piñas (Ananas spp.) (C). (Elaborada por JM España-UTadeo y E Barbosa - Agrosavia). 
Como se puede apreciar el proceso investigativo que se esta adelantando en el desarrollo de materiales compuestos con fibras vegetales y sus aplicaciones, es ambicioso y sus metas son a mediano plazo, parte de la intención de este proceso es lograr un programa de investigación que caracterice los materiales, a la vez que desde una fase mas aplicada y propia de las disciplinas del diseño se desarrollan los usos de los resultados obtenidos.

\section{Conclusiones}

- Este artículo busca mostrar a grandes rasgos un desarrollo investigativo y un interés, alrededor de un tema de gran potencial regional, a la vez que sirve como conducto de invitación a generar redes y sinergias en esta área investigativa. Es esencial que los investigadores latinoamericanos nos articulemos de manera dinámica, generosa y colaborativa, como estrategia para compensar otras debilidades de la región, como la falta de recursos financieros para la innovación e investigación aplicada.

- Las fibras naturales son de gran potencial en el desarrollo de productos que reemplacen a los plásticos, desde un menor impacto ambiental, pero se requiere más investigación en todos los niveles, para responder preguntas claves en el uso y aprovechamiento de las fibras naturales. ¿Cuáles fibras son potencialmente escalables a un mayor uso del recurso y bajo que condiciones, para evitar un deterioro ambiental? ¿Cómo protegemos nuestro patrimonio biótico mientras lo aprovechamos sosteniblemente? ¿Cómo garantizamos un beneficio socio-económico para los productores agrícolas de fibras vegetales?

- El desarrollo de biopolímeros y materiales compuestos con fibras vegetales debería apuntar a un desarrollo de productos y tecnologías locales, garantizando ciclos más sostenibles y dinámicos de la investigación.

- El desarrollo de materiales compuestos con petroplásticos y fibras naturales no tejidas y tejidas, debe apuntar a desplazar y desaparecer el uso de materiales como la fibra de vidrio, cuyo uso es muy perjudicial ambientalmente, pero con un uso muy extendido.

\section{Notas}

1. ACM hace alusión a Advanced composite materials.

2. Fique: (Furcraea ssp) Planta de cuyas hojas se extrae una fibra dura muy característica y utilizada en países andinos como Colombia y Ecuador.

3. Fique: (Furcraea ssp) Planta de cuyas hojas se extrae una fibra dura muy característica y utilizada en países andinos como Colombia y Ecuador.

4. Contenedor de base circular que se cuelga al hombro, muy representativo de las etnias indígenas de Colombia. Y cuyo uso, forma y simbolismo varía dependiendo del grupo indígena y su región.

5. INTI: Es el Instituto Nacional de Tecnología Industrial de Argentina. 
6. Corporación Colombiana de Investigación Agropecuaria, AGROSAVIA. es una entidad pública descentralizada de participación mixta sin ánimo de lucro, de carácter científico y técnico, cuyo objeto es desarrollar y ejecutar actividades de Investigación, Tecnología y transferir procesos de Innovación tecnológica al sector agropecuario. Extraído de: http:// www.corpoica.org.co/

\section{Referencias}

Abdellaoui, H.; Bensalah, H.; Echaabi, J.; Bouhfid, R. \& Qaiss, A. (2015). Fabrication, characterization and modelling of laminated composites based on woven jute fibres reinforced epoxy resin. Materials \& Design, 68, 104-113.

Ariza, R.; Benasso, T.; Dorado, C.; Flores, F.; Ramirez, R. y Yoguel, V. (2014). Objeto fieltro, oportunidades para agregar valor a la cadena lanera. Instituto nacional de tecnología industrial. Centro de investigación y desarrollo en diseño industrial. 152.

Asim, M.; Abdan, K.; Jawaid, M.; Nasir, M.; Dashtizadeh, Z.; Ishak, M. R. \& Hoque, M. E. (2015). A Review on Pineapple Leaves fibre and its composites. International Journal of Polymer Science. http://dx.doi.org/10.1155/2015/950567

ASTM (2007). Standard Test Method for Flexural Properties of Polymer Matrix Composite Materials. ASTM International, West Conshohocken, PA. doi: 10.1520/D7264_ D7264M-07

ASTM (2008). Standard test method for tensile properties of polymer matrix composite materials. ASTM International, West Conshohocken, PA. doi: 10.1520/D0638-08

ASTM (2010). Standard Test Method for Impact Resistance of Flat, Rigid Plastic Specimens by Means of a Falling Dart (Tup or Falling Mass). ASTM International, West Conshohocken, PA. doi: 10.1520/D5628-10

Bavan, D. S. \& Kumar, G. C. M. (2010). Potential use of natural fiber composite materials in India. Journal of Reinforced Plastics and Composites, 29(24): 3600-3613. https://doi. org/10.1177/0731684410381151

Bogoeva-Gaceva, G.; Avella, M.; Malinconico, M.; Buzarovska, A.; Grozdanov, A.; Gentile, G. \& Errico, M. E. (2007). Natural fiber eco-composites. Polym. Compos. 28: 98-107. doi:10.1002/pc.20270

Boujmal, R.; Essabir, H.; Nekhlaoui, S.; Bensalah, M. O.; Bouhfid, R. \& Qaiss, A. (2014). Composite from polypropylene and henna fiber: structural, mechanical and thermal properties. Journal of Biobased Materials and Bioenergy, 8(2), 246-252.

Castro, D.; Ruvolo-Filho, A. \& Frollini, E. (2012). Materials prepared from biopolyethylene and curaua fibers: Composites from biomass. Polymer Testing, 31(7), 880-888.

Chung, D. (2010). Composite materials: science and applications. Springer Science \& Business Media, 318.

España, J. M. y Barbosa, E. (Julio 2017). Las fibras naturales como foco de desarrollo sostenible en Latinoamérica. Desde la investigación transdisciplinar y sin fronteras. XI Convención Internacional sobre Medioambiente y Desarrollo Sostenible. Memorias. La Habana, Cuba, 910 pp. 
España, J. M. y Peña, V. (2013). Estrategia para el mejoramiento ambiental de las prácticas productivas y aumento de la productividad del fique en mercados verdes. Tesis de Grado. Maestría en Gestión Ambiental. Pontificia Universidad Javeriana. Bogotá. 136 pp.

España, J. M.; Rodriguez, J. y Romero, D. (2019). Experiencias pedagógicas que detonan oportunidades locales, Actio. Journal of technology in design, film arts and visual communication. 103-107.

FAO-CFC (2001). Alternative Applications for Sisal and Henequen. Proceedings of a Seminar held by the Food and Agriculture Organization of the UN (FAO) and the Common Fund for Commodities (CFC). Rome, 13 December 2000. Technical Paper No. 14. http:// www.fao.org/docrep/004/Y1873E/Y1873E00.HTM

Fowler, P. A.; Hughes, M. J. \& Elias, R. M. (2006). Review biocomposites: Technology, environmental credentials and market forces. J Sci Food Agric, 86, 1781-1789.

Gopinath, A.; Kumar, M. S. \& Elayaperumal, A. (2014). Experimental investigations on mechanical properties of jute fiber reinforced composites with polyester and epoxy resin matrices. Procedia Engineering, 97, 2052-2063.

Groover, M, (1997). Fundamentos de manufactura moderna: materiales, procesos y sistemas. Pearson Educación.

Hoyos, C. \& Vázquez, A. (2012). Flexural properties loss of unidirectional epoxy/fique composites immersed in water and alkaline medium for construction application. Composites Part B: Engineering, 43(8), 3120-3130.

Jaramillo, N.; Hoyos, D. y Santa, J. F. (2016). Composites with pineapple-leaf fibers manufactured by layered compression molding. Ingeniería y Competitividad, 18(2): 151-162.

John, M. J. \& Thomas, S. (2008). Biofibers and biocomposites. Carbohydr. Polym., 71(3), 343-364.

Joshi, S. V.; Drzal, L. T.; Mohanty, A. K. \& Arora, S. (2004). Are natural fiber composites environmentally superior to glass fiber reinforced composites? Composites Part A: Applied Science and Manufacturing, 35(3), 371-376.

Leao, A.; Souza, S.; Cherian, B.; Frollini, E.; Thomas, S.; Pothan, L. \& Kottai, S. (2010). Pineapple Leaf Fibers for Composites and Cellulose. Molecular Crystals and Liquid Crystals, 522, 336-341. DOI: 10.1080/15421401003722930.

Linares E. (1994). Inventario preliminar de las plantas utilizadas para elaborar artesanías en Colombia. Universitas Scientiarium, 2, 7-43.

Linares, E. L.; Figueroa, Y.; Galeano, G. y García, N. (2008). Fibras vegetales empleadas en artesanías en Colombia. Artesanías de Colombia S.A. Ministerio de Comercio, Industria y Turismo; Instituto de Ciencias Naturales Facultad de Ciencias- Universidad Nacional de Colombia. 333 pp.

Mohammed, L.; Ansari, M. N. M.; Pua, G.; Jawaid, M. e Islam, M. S. (2015). A Review on Natural Fiber Reinforced polymer composite and its applications. Int. J. Polymer Science. http://dx.doi.org/10.1155/2015/243947

Mohanty, A.; Misra, M. y Drzal, L. (2002). Sustainable bio-composites from renewable resources: opportunities and challenges in the green materials world. Journal of Polymers and the Environment, 10(1-2), 19-26.

Mohanty, A. K.; Misra, M. and Hinrichsen, G. (2000). Biofibres, biodegradable polymer and composites: an overview. Macromol Mater Eng, 276/277, 1-24. 
Moreno, G. (2017). Mechanical Properties Characterization of Advanced Composite Materials- Book Review. Ciencia y Poder Aéreo, 12(1), 288-290.

Neira-García, A.; Martínez-Reina, A. \& Orduz-Rodríguez, J. (2016). Análisis del mercado de piña Gold y Perolera en dos principales centrales mayoristas de Colombia. Corpoica Ciencia y Tecnología Agropecuaria, 17(2), 149-165.

Peréz A. Inventario crítico de las máquinas desfibradoras en México (1830-1890): una propuesta de documentación histórica y evaluación técnica para la investigación en diseño industrial. Universidad Autónoma Metropolitana, Unidad Azcapotzalco, 1999.

Sathish, T. \& Periyasamy, P. (2017). Checking the Mechanical Properties of Ananas comosus leaf fiber reinforced polymer composite material. International Journal of Pure and Applied Mathematics, 116(24), 243-253.

Saiter, J.; Dobircau, L. \& Leblanc, N. (2012). Are 100\% green composites and green thermoplastics the new materials for the future? International

Taj, S.; Ali, M. \& Khan, S. (2007).Natural fiber-reinforced polymer composites: review. Proc Pakistan Acad Sci, 44(2), 129-144.

Valero-Valdivieso, M.; Ortegón, Y. \& Uscategui, Y. (2013). Biopolímeros: avances y perspectivas. Dyna, 80(181), 171-180.

Wambua, P.; Ivens, J. \& Verpoest, I. (2003). Natural fibres: can they replace glass in fibre reinforced plastics? Compos Sci Technol, 63, 1259-1264.

Zah, R.; Hischier, R.; Leão, A. L.; Braun, I. (2007). Curauá fibers in the automobile industry-a sustainability assessment. Journal of Cleaner Production,15 (11-12), 1032-1040.

Abstract: Latin America is characterized by the biotic richness and biodiversity of its ecosystems that from pre-Hispanic times were the support of human settlements throughout the continent. This diversity favored the emergence of indigenous communities characterized by the territory, which provided them with what they needed for their sustenance and development. The vegetal fibers have been from those times a factor of identity and a reflection of material and immaterial cultural wealth. Ties, clothing, tools, backpacks and other objects of the indigenous ethnic groups and of the Latin American peasant culture are a reflection of knowledge. The invasion of polymers derived from petroleum relegated and threatened the continuity in the use of vegetable fibers. The plastic boots, the polypropylene ties, the mantles and the polyester fabrics put the vegetal fibers in the background for decades and caused the disappearance of ancestral knowledge manifested in utilitarian crafts. However, the threatening increase of global warming, the contamination of water resources with plastics and the enormous consequences in ecosystems due to our demand for oil and its derivatives, has led the world in the last decades to strengthen research and development in new materials and applications of vegetable fibers and by-products of agro-industry, to replace or minimize the use of plastics.

Now Latin America is one of the regions with the most bioprospecting potential in the use and exploitation of vegetable fibers, both because of the abundance and diversity it possesses and because of the knowledge and techniques that are linked to rural communities 
throughout the continent. However, it seems that we are lagging behind in the research around fibers and their potential in new materials and applications.

Which fibers are better in the development of composite materials? What are the advantages and environmental consequences of increasing their utilization? How does it benefit and / or affect the rural communities that produce these fibers? What are its physical and mechanical qualities? These are some of the many questions that we should ask from the investigation. And although there are several research processes in the academy in this regard, the review of indexed articles and academic publications seems to suggest that it is other regions such as Europe, North America and Asia that are taking the lead in the research, use and application of fibers in new materials and products, despite not having a biodiversity like the Latin American one. At the Jorge Tadeo Lozano University in Bogotá, from the School of Product Design we are focused on investigating the use of vegetable fibers and by-products of agro-industry for the development of new materials and applications. Design had a very important role in the mass use of plastics, now must be an active part in the search for alternative materials that enhance the sustainable use of resources and the strengthening of product developments for local economies. The vegetal fibers were in the past the cradle of local knowledge to solve the daily and rural needs, it is time that we return to this route. A group of researchers made up of undergraduate and postgraduate professors and students, in partnership with agricultural research institutions and fiber producing communities, are in search of developing new composite materials (ACM) biopolymers and local technological transformation systems, to encourage the use and application of local materials in the development of products capable of competing with those made of plastic materials, but with all the advantages implied by the use of biodegradable and sustainable materials. This article describes the route that we as researchers are traveling and invites peers from other latitudes to join us in this effort.

Keywords: Vegetable fibers - Sustainability - Plastic Materials - Ancestral knowledge Technological development - New materials - ACM. (Advanced composite materials).

Resumo: A América Latina é caracterizada pela riqueza biótica e biodiversidade de seus ecossistemas que, desde a época pré-hispânica, eram o suporte de assentamentos humanos em todo o continente. Essa diversidade favoreceu o surgimento de comunidades indígenas caracterizadas pelo território, o que lhes proporcionou o que elas necessitavam para seu sustento e desenvolvimento. As fibras vegetais foram desde então um fator de identidade e um reflexo da riqueza cultural material e imaterial. Laços, roupas, ferramentas, mochilas e outros objetos das etnias indígenas e da cultura camponesa latino-americana são reflexos do conhecimento. A invasão de polímeros derivados do petróleo relegou e ameaçou a continuidade no uso de fibras vegetais. As botas plásticas, os laços de polipropileno, os mantos e os tecidos de poliéster colocaram as fibras vegetais em segundo plano por décadas e provocaram o desaparecimento do conhecimento ancestral manifestado nos ofícios utilitários.

No entanto, o aumento ameaçador do aquecimento global, a contaminação dos recursos hídricos com os plásticos e as enormes consequências nos ecossistemas devido à nossa demanda por petróleo e seus derivados, levaram o mundo nas últimas décadas a fortalecer 
a pesquisa e o desenvolvimento em novos materiais e aplicações de fibras vegetais e subprodutos da agroindústria, para substituir ou minimizar o uso de plásticos.

Atualmente, a América Latina é uma das regiões com maior potencial de bioprospecção no uso e aproveitamento das fibras vegetais, tanto pela abundância e diversidade que possui, como pelos conhecimentos, técnicas e conhecimentos que estão vinculados às comunidades rurais de todo o continente. No entanto, parece que estamos ficando para trás na pesquisa em torno de fibras e seu potencial em novos materiais e aplicações.

Quais fibras são melhores no desenvolvimento de materiais compostos? Quais são as vantagens e consequências ambientais de aumentar seu uso e utilização? Como isso beneficia e / ou afeta as comunidades rurais que produzem essas fibras? Quais são as suas qualidades físicas e mecânicas? Estas são algumas das muitas perguntas que devemos fazer desde a investigação pesquisar. E embora existam vários processos de pesquisa na academia nesse sentido, a revisão de artigos indexados e publicações acadêmicas parece sugerir que outras regiões, como Europa, América do Norte e Ásia, estão liderando a pesquisa, uso e aplicação de fibras, novos materiais e produtos, apesar de não ter uma biodiversidade como a latino-americana. Na Universidade Jorge Tadeo Lozano em Bogotá, da Escola de Design de Produto, estamos focados em investigar o uso de fibras vegetais e subprodutos da agroindústria para o desenvolvimento de novos materiais e aplicações.

O projeto teve um papel muito importante no uso em massa de plásticos, agora deve ser um componente ativo na procura de materiais alternativos que melhorem o uso sustentável dos recursos e o fortalecimento do desenvolvimento de produtos para as economias locais. As fibras vegetais foram no passado o berço do conhecimento local para resolver as necessidades diárias e rurais, é hora de voltarmos a essa rota. Um grupo de pesquisadores composta por professores e alunos de graduação e pós-graduação, em parceria com instituições de pesquisa agrícola e agricultura fibras comunidades estão em busca do desenvolvimento de novos materiais compósitos biopolímeros e sistemas de transformação tecnológica local (ACM), para incentivar uso e aplicação de materiais locais no desenvolvimento de produtos capazes de competir com aqueles feitos de materiais plásticos, mas com todas as vantagens implicadas pelo uso de materiais biodegradáveis e sustentáveis. Este artigo descreve a rota que nós, pesquisadores, estamos percorrendo e convida casais de outras latitudes para fazer contato com esse esforço.

Palavras chave: fibras vegetais - sustentabilidade - materiais plásticos - saberes ancestrais - desenvolvimento tecnológico - novos materiais - ACM. 